\begin{tabular}{l}
\hline OPEN O ACCESS Freely available online \\
http://www.banglajol.info/index.php/BJID/index \\
Original Article \\
Bangladesh Journal of Infectious Diseases \\
December 2016, Volume 3, Number 2 \\
ISSN (Online) 2411-670X \\
ISSN (Print) 2411-4820
\end{tabular}

\title{
Histopathological Features of Lymph Nodes of Tuberculous Lymphadenitis Patients: Experience of 50 cases in Bangladesh
}

\author{
Mohammad Mahmudul Huda ${ }^{1}$, Mohammad Taufiq ${ }^{2}$, Md. Abdullah Yusuf ${ }^{3}$, Mohammad Rezwanur \\ Rahman $^{4}$, Ferdousy Begum $^{5}$, Mohammed Kamal ${ }^{6}$
}

${ }^{1}$ Assistant Professor, Department of Pathology, Dhaka National Medical College, Dhaka, Bangladesh; ${ }^{2}$ Associate Consultant (Pathology), Square Hospital, Dhaka, Bangladesh; ${ }^{3}$ Assistant Professor, Department of Microbiology, National Institute of Neurosciences \& Hospital, Dhaka, Bangladesh; ${ }^{4}$ Professor, Department of Biochemistry, Delta Medical College, Dhaka, Bangladesh; ${ }^{5}$ Associate Professor, Department of Pathology, Banghabandhu Sheikh Mujib Medical University, Dhaka, Bangladesh; ${ }^{6}$ Professor, Department of Pathology, Banghabandhu Sheikh Mujib Medical University, Dhaka, Bangladesh

[Received: 10January 2016; Revised: 12March 2016; Accepted:26 October 2016; Published:1 December 2016]

\begin{abstract}
Background: Tuberculous lymphadenitis is common manifestation of extra-pulmonary tuberculosis in Bangladesh. Objective: The purpose of the present study was to see the histopathological features of tuberculous lymphadenitis patients. Methodology: This cross sectional study was done at the Department of Pathology at Banghabandhu Sheikh Mujib Medical University (BSMMU), Dhaka from January 2009 to March 2011 for a period of nearly two years. All the patients irrespective of age and sex with the clinical features suggestive of tuberculous lymphadenitis and later on proved to be the same histologically were selected for the study purposively. Relevant information was recorded in a prescribed data sheet and histomorphological findings were recorded accordingly. In cases where fresh specimen was available, caseous portion of lymph node was sent for culture. FiteFaraco staining was also done on lymph node sections in all cases. Result: A total number of 50 tuberculous lymphadenitis patients were recruited for this study of which well formed granuloma was detected in 34(68.0\%) cases followed by ill defined and combination of well and ill defined which were 8(16.0\%) cases in each. Among 34 well defined cases coalescing and discrete were found in 6(17.6\%) cases in each. However, both types were found in $22(64.7 \%)$ cases.Conclusion: In conclusion well formed granuloma is most commonly found in tuberculous lymphadenitis with combined variety of coalescing and discrete histopathologic features.[Bangladesh Journal of Infectious Diseases 2016;3(2):40-42]

Keywords: Tuberculous lymphadenitis; tuberculosis; lymph nodes; histopathological features

Correspondence:Dr. Mohammad Mahmudul Huda, Assistant Professor, Department of Pathology, Dhaka National Medical College, Dhaka, Bangladesh; Email: mmhuda.himel@gmail.com ; Cell no.: +8801819203320

Conflict of interest: There is no conflict of interest to any of the authors of this article.

Funding agency: This research work was performed by own cost. There was funding agency had given the support.

Contribution to authors: MMH, MT, MRR, FB\&MK were involved from protocol preparation, data collection, data analysis up to report writing. MMH \& MAY prepared and revised the manuscript.

How to cite this article:Huda MM, Taufiq M, Yusuf MA, Rahman MR, Begum F, Kamal M. Histopathological Features of Lymph Nodes of Tuberculous Lymphadenitis Patients: Experience of 50 cases in Bangladesh. Bangladesh Journal of Infectious Diseases 2016;3(2):40-42
\end{abstract}




\section{Introduction}

Lymph nodes are usually involved in the early stages of the pulmonary disease or as secondary TB by hematogenous spread ${ }^{1}$. However, tuberculous lymphadenitis may arise without a detectable preceding pulmonary involvement ${ }^{2}$. Tuberculous lymphadenitis affects mainly the cervical lymph node group and is an important cause of lymphadenopathy worldwide ${ }^{3}$.

The clinical as well as the demographic characteristics are varied. To confirm the cases histopathological examination or FNAC is needed. These help to arrive at an early diagnosis of tubercular lymphadenitis and institution of treatment before a final diagnosis is made by culture $^{4}$. Histopathological examination is suggestive of tuberculous lymphadenitis where Langhans' giant cells, caseation necrosis, coalescing granuloma are present. The physicians treat these cases with anti-tubercular chemotherapy.

In cases which are reported as 'suggestive of tuberculosis', the physician needs additional features such as positive Mantoux test and clinical symptoms to start anti-tubercular chemotherapy. Therefore the clinical as well as the demographics profiles are important for the treatment of tuberculous lymphadenitis. In this context the present study was undertaken to see the histopathological features of tuberculous lymphadenitis patients.

\section{Methodology}

This cross sectional study was done at the Department of Pathology at Banghabandhu Sheikh Mujib Medical University (BSMMU), Dhakafrom January 2009 to March 2011 for a period of nearly two years. All the patients irrespective of age and sexwith the clinical features suggestive of tuberculous lymphadenitis and later on proved to be the samehistologically were selected for the study purposively. The patients without having history of anti-tubercular drugs, malignancy and symptoms other than TB were excluded from this study. Relevant informationwas recorded in a prescribed data sheet and histomorphological findings were recorded accordingly.In cases where fresh specimen was available, caseous portion of lymph node was sent to ICDDRB for culture in conventional egg based Lowenstein- Jensen medium. This part was done maintaining high level of sterility.ZiehlNeelsen stain was done in smear prepared from 20 fresh cases.FiteFaraco staining was also done on lymph node sections in all cases.Computer based statistical analysis was carried out with appropriate Bangladesh J Infect Dis techniques and systems. All data were recorded systematically in preformed data collection form (questionnaire) and quantitative data were expressed as mean and standard deviation and qualitative data were expressed as frequency distribution and percentage. Statistical analysis was performed by using window based computer software devised with Statistical Packages for Social Sciences (SPSS-22.0) (SPSS Inc, Chicago, IL, USA). 95\% confidence limit was taken. The summarized data was interpreted accordingly and was then presented in the form of tables figures.

\section{Results}

A total number of 50 tuberculous lymphadenitis patients were recruited for this study.Out of 50 cases well formed granuloma was detected in $34(68.0 \%)$ cases followed by ill defined and combination of well and ill defined which were $8(16.0 \%)$ cases in each. Among 34 well defined cases coalescing and discrete were found in $6(17.6 \%)$ cases in each. However both types were found in $22(64.7 \%$ ) cases (Table 1$)$.

Table 1: Types of granuloma Among the Study Population

\begin{tabular}{ccc}
\hline Types of Granuloma & Frequency & Percentage \\
\hline Well formed & 34 & 68.0 \\
$\bullet$ Coalescing & 06 & 17.6 \\
$\bullet$ Discrete & 06 & 17.6 \\
• Both & 22 & 64.7 \\
Ill defined & 8 & 16.0 \\
Combined Form & 8 & 16.0 \\
\hline
\end{tabular}

Ten patients (20\%) caseshad single lymphadenopathy and the rest $40(80 \%)$ cases had multiple lymphadenopathyand $8(16 \%)$ patients had more than one group of lymphadenopathy in single region (Table2).

Table 2: Relation between Number of Lymph Nodes and Types of Granuloma

\begin{tabular}{lcccc}
\hline \multirow{2}{*}{$\begin{array}{l}\text { Lymph } \\
\text { node }\end{array}$} & \multicolumn{2}{c}{ Types of Granuloma } & Total \\
\cline { 2 - 4 } & $\begin{array}{l}\text { Well } \\
\text { defined }\end{array}$ & $\begin{array}{l}\text { Ill } \\
\text { defined }\end{array}$ & Both & \\
\hline Single & 4 & 3 & 3 & 10 \\
Multiple & 30 & 4 & 6 & 40 \\
Total & $\mathbf{3 4}$ & $\mathbf{7}$ & $\mathbf{9}$ & $\mathbf{5 0}$ \\
\hline
\end{tabular}

\section{Discussion}

Someone in the world is newly infected with TB bacilli every second. Overall, one-third of the world's population is currently infected with the TB bacillus ${ }^{5}$. Among the various diagnostic tools of December 2016 | Volume 3 | Number 2 
tubercular lymphadenitis histological diagnosis is an important one. As the morphological features are variable this descriptive study was performed in $\mathbf{5 0}$ cases of tubercular lymphadenitis to find out histomorphological features and correlate with clinical profile and treatment outcome.

Histomorphology shows well formed granuloma in $68 \%$ of cases. Among the well formed granuloma $64.7 \%$ had both coalescing and discrete granuloma, $17.6 \%$ had coalescing granuloma and $17.6 \%$ had only discrete granuloma. In another study it has been found that well formed granuloma is detected in $89 \%$ cases which is higher than this present study. Extent of caseous necrosis is also observed in present study. Of 50 patients, $18 \%$ patient had focal area of caseous necrosis, $54 \%$ had moderate caseous necrosis and $28 \%$ had extensive caseous necrosis. This present study showed variation in percentage of caseous necrosis in individual cases.

All patients were treated with 6 months chemo regimen comprising of four drugs like rifampicin, isoniazid, Ethambutol and pyrazinamide for initial two months followed by rifampicin and isoniazid for next four months. These drugs are taken daily. After six months of treatment every patient was assessed by treating physician. Chemotherapy was extended up to nine months in $10(20 \%)$ cases. On clinical ground $1(2 \%)$ patient needed to extend six months more as the physician assessed the treatment was not responding. Thirty six patients (72\%) completed six months treatment with disappearance of constitutional symptoms including fever, weight loss and cough along with disappearance of lymphadenopathy.

Although pulmonary TB represents a major health problem, tuberculosis lymphadenitis represents another extra burden $^{6-8}$. Conventional $\mathrm{ZN}$ and fluoresces methods for tuberculosis have several limitations and are often unhelpful in establishing the diagnosis of lymph node tuberculosis. Histopathology remains one of the most important methods for diagnosing tuberculosis, and in a high TB prevalent area histopathology is the reliable and a gold standard as otherwise is the culture. However, it cannot differentiate changes caused by M. tuberculosis, non-tuberculous mycobacterium, or other granulomatous diseases ${ }^{7}$.
In endemic countries, the majority of granulomatous lesions without necrosis are considered to be tuberculosis, but this may not be the case in the developed world ${ }^{8}$. Therefore, it is advisable to use more specific and sensitive methods, such as, PCR or immune-histochemistry, before reporting the negative results.

\section{Conclusion}

In conclusion well formed granuloma is most commonly found in tuberculous lymphadenitis with combined variety of coalescing and discretehistopathologic features.Further large scale multicentrestudy should be carried out to get the accurate pictures of the histopathologic findings oftuberculous lymphadenitis.

\section{References}

1. Murray CJ, Styblo K, Rouillon A. Tuberculosis in developing countries: burden, intervention and cost. Bulletin of International Union against Tuberculosis and Lung Disease 1990;65(1):6-24

2. Kumar V, Abbas AK, Fausto N, Aster JC. Robbins and CotranPathologic Basis of Disease. Elsevier Health Sciences; 2014 Aug 27

3. Thompson MM, Underwood MJ, Sayers RD, Dookeran KA, Bell PR. Peripheral tuberculous lymphadenopathy: a review of 67 cases. British Journal of Surgery1992;79(8):763-4

4. Lau SK, Wei WI, Hsu C, Engzell UC. Efficacy of fine needle aspiration cytology in the diagnosis of tuberculous cervical lymphadenopathy. The Journal of Laryngology \& Otology 1990;104(1):24-7

5. Ahmed HG, Nassar AS, Ginawi I. Screening for tuberculosis and its histological pattern in patients with enlarged lymph node. Pathology Research International. 2011;2011

6. Dandapat MC, Mishra BM, Dash SP, Kar PK. Peripheral lymph node tuberculosis: a review of 80 cases. British Journal of Surgery 1990;77(8):911-2

7. Moore SW, Schneider JW, Schaaf HS. Diagnostic aspects of cervical lymphadenopathy in children in the developing world: a study of 1,877 surgical specimens. Pediatric Surgery International 2003;19(4):240-4

8. Marais BJ, Wright CA, Schaaf HS, Gie RP, Hesseling AC, Enarson DA, Beyers N. Tuberculous lymphadenitis as a cause of persistent cervical lymphadenopathy in children from a tuberculosis-endemic area. The Pediatric Infectious Disease Journal 2006;25(2):142-6 